\title{
Upregulation of BTG1 enhances the radiation sensitivity of human breast cancer in vitro and in vivo
}

\author{
RAN ZHU $^{1 *}$, WEI LI $^{2 *}$, YAN XU $^{3}$, JIANMEI WAN ${ }^{1}$ and ZENGLI ZHANG ${ }^{4}$ \\ ${ }^{1}$ Jiangsu Provincial Key Laboratory of Radiation Medicine and Protection, School of Radiation Medicine and Protection, \\ Medical College of Soochow University, Collaborative Innovation Center of Radiation Medicine, \\ Jiangsu Higher Education Institutions; ${ }^{2}$ Department of General Surgery, Second Affiliated Hospital of \\ Soochow University; ${ }^{3}$ Department of General Surgery, First Affiliated Hospital of Soochow University; \\ ${ }^{4}$ Jiangsu Key Laboratory of Preventive and Translational Medicine for Geriatric Diseases, \\ School of Public Health, Soochow University, Suzhou, Jiangsu, P.R. China
}

Received July 3, 2015; Accepted August 4, 2015

DOI: 10.3892/or.2015.4311

\begin{abstract}
X-ray-based radiotherapy is one of the most effective therapeutic strategies for breast cancer patients. However, radioresistance and side-effects continue to be the most challenging issues. B-cell translocation gene 1 (BTG1) is a member of the BTG/Tob family, which inhibits cancer growth and promotes apoptosis. We, therefore, hypothesized that BTG1 plays an important role in the radiosensitivity of breast cancer cells. In the present study, breast cancer cell lines that stably overexpressed BTG1 were used to investigate the effects of BTG1 on cell radiosensitivity in vitro. We found that overexpression of BTG1 enhanced the radiosensitivity both of p53-mutant breast cancer MDA-MB-231 cells and p53 wild-type breast cancer MCF-7 cells. We also found that overexpression of BTG1 along with irradiation induced cell cycle $\mathrm{G} 2 / \mathrm{M}$ phase arrest, promoted the formation of reactive oxygen species (ROS), increased the rate of chromosomal aberrations and increased cell apoptosis. Further investigation indicated that BTG1 overexpression along with irradiation was involved in inhibition of the PI3K/Akt signaling pathway. Importantly, the finding that BTG1 promoted ionizing radiosensitivity of breast cancer cells in vitro was confirmed in an animal model. Taken together, our data suggest that BTG1 overexpression combined with radiation therapy increases the therapeutic efficacy of breast cancer treatment via regulation of the cell cycle and apoptosis-related signaling pathways.
\end{abstract}

Correspondence to: Professor Zengli Zhang, Jiangsu Key Laboratory of Preventive and Translational Medicine for Geriatric Diseases, School of Public Health, Soochow University, 199 Ren'ai Road, Suzhou Industrial Park, Suzhou, Jiangsu 215123, P.R. China E-mail: qhk77@sina.com

*Contributed equally

Key words: breast cancer, BTG1, radiosensitivity, cell cycle, apoptosis

\section{Introduction}

Breast cancer is the most common female cancer and one of the leading causes of cancer-related deaths worldwide with a relatively high incidence rate $(1,2)$. Surgery, chemotherapy and radiotherapy are the traditional therapeutic methods for the treatment of breast cancer, and radiotherapy is an important adjuvant therapy for breast cancer patients (3).

However, resistance to radiotherapy often results in treatment failure, particularly for loco-regional recurrence. Tumor-suppressor genes that regulate the cell cycle and induce cell apoptosis can reverse the radiation resistance of cancer cells $(4,5)$.

B-cell translocation gene 1 (BTG1) is a tumor-suppressor gene, and belongs to the antiproliferative gene family comprising pheochromacytoma cell-3, tetradecanoyl phorbol acetate-inducible sequence 21, BTG3, transducer of ERBB2, 1 (TOB1) and TOB2 (6,7). Proteins encoded by members of this gene family induce growth arrest or apoptosis in a variety of cell systems (8). Overexpression of BTG1 has been found to inhibit proliferation during normal erythroid differentiation (9) and induce cell cycle arrest or apoptosis in several cell types, including NIH3T3 murine fibroblasts (10), myoblasts (11) and microglia (12). BTG1 is also essentially expressed in many types of tumor cells and inhibits the proliferation of a variety of cancer cells. Recent studies have demonstrated that BTG1 protein levels are significantly reduced in breast cancer and are associated with the pathogenesis and progression of breast carcinomas (13). Overexpression of BTG1 inhibited breast cancer cell invasion and metastasis both in vitro and in vivo (14). Downregulation of BTG1 by miR454-3p enhanced cellular radiosensitivity in renal carcinoma cells due to the role of BTG1 in cell cycle progression (15). Importantly, we reported that in breast cancer cells, BTG1 inhibits cell growth through induction of cell cycle arrest and apoptosis (16). We, therefore, hypothesized that BTG1 plays an important role in the radiosensitivity of breast cancer cells. Thus, the present study was undertaken to evaluate the effect of BTG1 along with X-ray irradiation on breast cancer cell (MDA-MB-231 and MCF-7) colony formation, cell cycle 
distribution and apoptosis in vitro. Furthermore, we examined whether these changes are also observed in vivo using a nude mouse model.

\section{Materials and methods}

Cell culture. Human breast cancer MCF-7 and MDA-MB-231 cell lines were obtained from the Shanghai Cell Bank (Shanghai, China). The stable cell lines MDA-MB-231/BTG1, MDA-MB-231/Neo, MCF-7/BTG1 and MCF-7/Neo which overexpressed BTG1 and control cell lines were preserved in our laboratory (16), and were maintained in Dulbecco's modified Eagle's medium (DMEM) (Gibco-BRL, USA) supplemented with $10 \%$ fetal bovine serum (FBS) and were cultured in an incubator at $37^{\circ} \mathrm{C}$ with $5 \% \mathrm{CO}_{2}$.

Irradiation. The cells were irradiated with different doses of $0,0.5,1,3,6$ and 9 Gy rays using a Primus High-Energy Siemens irradiator at a dose rate of $200 \mathrm{cGy} / \mathrm{min}$ at room temperature.

Colony formation assay. For the colony formation assay, transfected overexpression and control cells were plated in $60-\mathrm{mm}$ culture dishes at a cell density of $2 \times 10^{3}$ for $24 \mathrm{~h}$. Cells were exposed to $0,0.5,1,3,6$ and 9 Gy doses of irradiation and then incubated at $37^{\circ} \mathrm{C}$ for 2 weeks post-irradiation. Cells were fixed with methanol and stained with Giemsa solution. Colonies containing $>50$ cells were counted. Colony forming efficiency $=$ number of colonies formed $/$ number of cells plated. A multi-target click model $\left[\mathrm{S}=1-\left(1-\mathrm{e}^{-\mathrm{D} / \mathrm{D} 0}\right)^{\mathrm{N}}\right]$ was applied to delineate the survival curve; D0, lethal dose. Then, Dq (quasi-threshold dose) $=$ D0 x LN (N) was calculated.

Flow cytometric analysis of cell cycle distribution and apoptosis. For the cell cycle assay, transfected overexpression and control cells were plated into 6-well plates at a cell density of $5 \times 10^{5}$ and allowed to attach overnight. Cells were exposed to 0 , $1,3,6$ and 9 Gy doses of radiation, incubated at $37^{\circ} \mathrm{C}$ for $24 \mathrm{~h}$ post-irradiation and then fixed with $70 \%$ ice-cold ethanol at $4^{\circ} \mathrm{C}$ overnight. Fixed cells were washed with PBS and stained with propidium iodide $(100 \mu \mathrm{g} / \mathrm{ml})$ for $30 \mathrm{~min}$ in the dark before analysis. The cell cycle profiles were assayed using the FACSCan ESP flow cytometer at $488 \mathrm{~nm}$, and data were analyzed using MultiCycle software (BD Biosciences, USA). For analysis of apoptosis, cells were exposed to a $3 \mathrm{~Gy}$ dose of irradiation and were then incubated at $37^{\circ} \mathrm{C}$ for $24 \mathrm{~h}$ post-irradiation and processed as described in the Annexin V-FITC apoptosis detection kit (BD Biosciences) and analyzed on a FC500 flow cytometer.

Western blot analysis. Protein concentration was determined with a protein assay. Equal amounts of protein were separated using 10\% SDS-PAGE gel electrophoresis, transferred onto nitrocellulose membranes and blocked with 5\% skimmed milk. Following blocking, the membranes were incubated with antibodies against $\beta$-actin, cyclin B1, p-p53, AKT, p-AKT, Bcl-2 and Bax and were then incubated with HRP-conjugated anti-mouse or anti-rabbit IgG antibodies (both from Santa Cruz Biotechnology, Santa Cruz, CA, USA). Protein bands were visualized with ECL solution.
DCFH-DA fluorescence probe analysis of reactive oxygen species (ROS). Transfected overexpression and control cells were plated into 6-well plates at a cell density of $1 \times 10^{5}$ and allowed to attach overnight. Cells were exposed to a $3 \mathrm{~Gy}$ dose of irradiation and were then incubated at $37^{\circ} \mathrm{C}$ for $0.5,1,2,4$ and $24 \mathrm{~h}$ post-irradiation. Cells were harvested, resuspended in $100 \mu \mathrm{l}$ PBS and $50 \mu \mathrm{l}$ chloromethyl-2',7'-dichlorofluorescein diacetate (50 $\mu \mathrm{M}$ DCFH-DA) was added for $15 \mathrm{~min}$ at $37^{\circ} \mathrm{C}$ in the dark. Then the cells were washed with PBS and treated with $1 \mathrm{mg} / \mathrm{l} \mathrm{ROS}$ for up to $15 \mathrm{~min}$ at $37^{\circ} \mathrm{C}$ in the dark. The intensity of fluorescence was assayed using the FC500 flow cytometer at $488 \mathrm{~nm}$.

Chromosomal aberration analysis. Exponentially growing transfected overexpression and control cells were exposed to 0 , 1,3,6 and 9 Gy doses of irradiation and were then treated with $400 \mathrm{ng} / \mathrm{ml}$ colchicine at $37^{\circ} \mathrm{C}$ for $8 \mathrm{~h}$ post-irradiation. Cells were harvested, administered hypotonic treatment of $0.075 \mathrm{M}$ $\mathrm{KCl}$, were fixed onto a slide glass with methanol:acetic acid (3:1) for two times and stained with $10 \%$ Giemsa. Chromosome aberrations including dicentric (dic) and centromere ring (r) were counted by microscopic examination.

In vivo studies. The nude mouse models of breast cancer were constructed using MDA-MB-231, MDA-MB-231/Neo and MDA-MB-231/BTG1 cells as previously described (16). Each group consisted of 12 nude mice, and 6 nude mice were randomly selected to accept a 3 Gy dose of irradiation. Every four days, the tumor diameter was measured, and the volume was calculated according to the formula: $\mathrm{V}=0.4 \mathrm{x}$ largest diameter $\mathrm{x}$ smallest diameter. Four weeks after injection of the cells, the mice were sacrificed and the tumors were separated. Tissue sections were deparaffinized, rehydrated and rinsed, prior to hematoxylin and eosin (H\&E) staining and examination for metastatic nodules and cell pathology. The animal treatment protocol used in the present study was approved by the Institutional Animal Care and Use Committee.

Immunohistochemistry. Tumor tissue sections were treated with $0.03 \%$ hydrogen peroxide for $5 \mathrm{~min}$ to block endogenous peroxidase activity and were then incubated with CD31, vascular endothelial growth factor (VEGF) and Bcl-2 antibodies (Santa Cruz Biotechnology) diluted at 1:100 for $60 \mathrm{~min}$ at room temperature. Following washing in PBS, the sections were incubated with labeled HRP-conjugated anti-mouse antibody, for $30 \mathrm{~min}$ at room temperature, prior to washing twice in PBS and incubating with diaminobenzene for $10 \mathrm{~min}$. Following washing, the sections were counterstained with hematoxylin, washed and dipped briefly in a water bath containing drops of ammonia, prior to dehydration and mounting in Diatex. The stained sections were analyzed and scored using a Nikon microscope (Nikon Corporation, Japan).

TUNEL staining assay. Terminal deoxynucleotidyl transferasemediated dUTP-biotin nick-end labeling (TUNEL) assay was performed using recombinant terminal transferase (TdT) and biotin-16-dUTP (Sigma, USA). Tumor tissue sections were processed following the manufacturer's protocol, and then the stained sections were reviewed and scored using a Nikon microscope. 

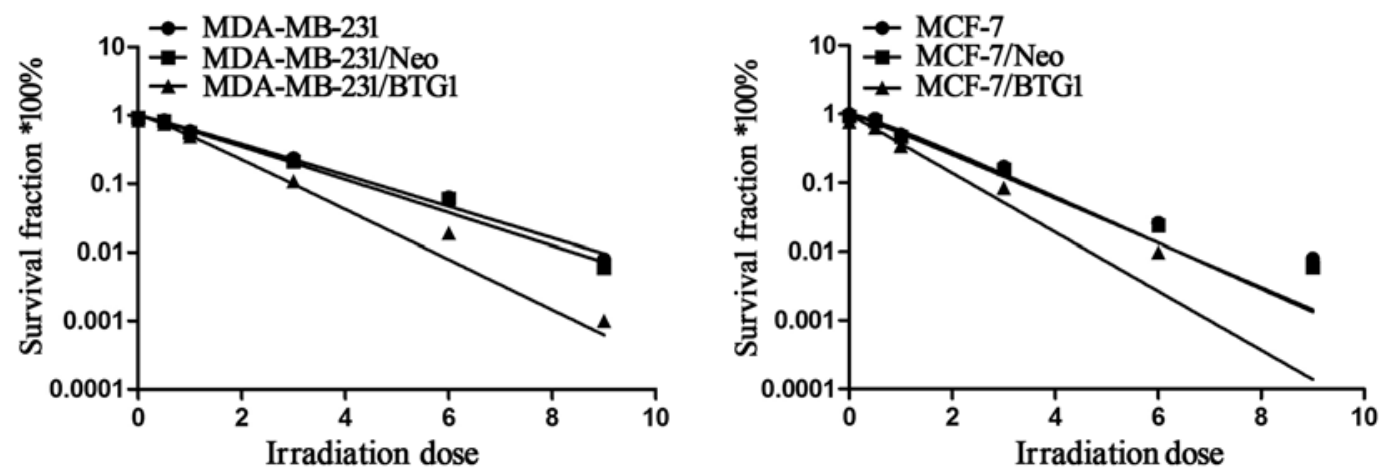

Figure 1. Clonogenic survival of the MDA-MB-231 and MCF-7 cells two weeks post irradiation. All the cell groups received 0-9 Gy of irradiation and colony formation was detected two weeks after irradiation.

Table I. Relative biological parameters of the different cell groups after calculation using the multi-target single-hit model.

\begin{tabular}{lcccc}
\hline Cell group & Fitting curve equation & $\mathrm{D}_{0}$ & $\mathrm{D}_{\mathrm{q}}$ & $\mathrm{N}$ \\
\hline MDA-MB-231 & $\mathrm{SF}=1-\left(1-\mathrm{e}^{-0.5683 \mathrm{D}}\right)^{1.41}$ & 1.76 & 1.61 & 1.41 \\
MDA-MB-231/Neo & $\mathrm{SF}=1-\left(1-\mathrm{e}^{-0.5933 \mathrm{D}}\right)^{1.34}$ & 1.69 & 0.49 & 1.34 \\
MDA-MB-231/BTG1 & $\mathrm{SF}=1-\left(1-\mathrm{e}^{-0.7739 \mathrm{D}}\right)^{1.33}$ & 1.29 & 0.37 & 1.33 \\
MCF-7 & $\mathrm{SF}=1-\left(1-\mathrm{e}^{-0.5694 \mathrm{D}}\right)^{1.74}$ & 1.79 & 0.99 & 1.74 \\
MCF-7/Neo & $\mathrm{SF}=1-\left(1-\mathrm{e}^{-0.56790 \mathrm{D}}\right)^{1.61}$ & 1.79 & 0.86 & 1.61 \\
MCF-7/BTG1 & $\mathrm{SF}=1-\left(1-\mathrm{e}^{-0.7554 \mathrm{D}}\right)^{1.19}$ & 1.32 & 0.22 & 1.19 \\
\hline
\end{tabular}

Statistical analysis. Statistical analyses were carried out using SPSS 17.0. Each experiment was repeated 3 times. The results shown are the means $\pm \mathrm{SD}$. A p-value of $<0.05$ was considered to indicate a statistically significant difference.

\section{Results}

Effects of BTGI on the radiosensitivity of breast cancer cells. Each group of cells was irradiated at different doses $(0,0.5,1,3,6$ and $9 \mathrm{~Gy})$, respectively. After two weeks of incubation, clonogenic survival of each group was assessed. The cell survival curves were characterized according to the multi-target click model. As shown in Fig. 1, the cell survival curve of BTG1 overexpression cells shifted to the left both in the p53-mutant cells (MDA-MB-231) and in the p53 wildtype cells (MCF-7), indicating that cell radiosensitivity was increased. Table I shows the values of several radiobiological parameters by the multi-target model. The D0 values for the MDA-MB-231 and MDA-MB-231/Neo cells were 1.76 and $1.69 \mathrm{~Gy}$, yet the D0 value for the MDA-MB-231/BTG1 cells was reduced to $1.29 \mathrm{~Gy}$. Thus, the radiosensitization ratios were 1.36 and 1.31 , which indicated that the radiosensitivity of the MDA-MB-231/BTG1 cells was increased by 1.36 and 1.31. The same increasing trend was also observed in the MCF-7 cells. The D0 value for both MCF-7 and MCF-7/Neo cells was $1.79 \mathrm{~Gy}$, yet the D0 value for the MCF-7/BTG1 cells was $1.32 \mathrm{~Gy}$. Thus, the radiosensitization ratio was 1.36 , which indicated that the radiosensitivity of MCF-7/BTG1 cells was increased by 1.36 . These data indicate that overexpression of BTG1 enhanced the radiosensitivity of both the p53-mutant breast cancer MDA-MB-231 cells and the p53 wild-type breast cancer MCF-7 cells in vitro.

Effects of BTG1 along with irradiation on the cell cycle distribution of MDA-MB-231 and MCF-7 cells. The cells were irradiated at different doses of 0, 1, 3, 6 and $9 \mathrm{~Gy}$ and were then incubated at $37^{\circ} \mathrm{C}$ for $24 \mathrm{~h}$ post-irradiation. Flow cytometry was used to analysis the cell cycle distribution. As shown in Fig. 2A, with an increase in radiation dose, all groups of cells underwent G2 phase arrest and the apoptosis rate was also increased $(\mathrm{p}<0.05)$. In addition, the BTG1 overexpression group cells (MDA-MB-231/BTG1 and MCF-7/BTG1) had significantly increased $\mathrm{G} 2 / \mathrm{M}$ phase arrest $(\mathrm{p}<0.05)$ when compared with the control group cells.

Next, we evaluated the effects of BTG1 on the expression of cell cycle proteins cyclin B1 and p-p53 in the MDA-MB-231 and MCF-7 cells using western blot analysis. As shown in Fig. 2B, overexpression of BTG1 along with irradiation treatment led to a significant inhibition of cyclin B1 and promotion of p-p53. In summary, these results revealed that after breast cancer cells underwent DNA damage caused by irradiation, an increase in the level of BTG1 expression inhibited cyclin B1 expression and promoted p-p53 expression, which in turn affected the cell cycle distribution of the breast cancer cells (MDA-MB-231 and MCF-7) and induced G2/M phase arrest.

Effects of BTG1 along with irradiation on the apoptosis of MDA-MB-231 and MCF-7 cells. Annexin V staining was used to detect cell apoptosis. As determined by the Annexin $\mathrm{V}$ assay, in the MCF-7/BTG1 and MDA-MB-231/BTG1 cells the 
A

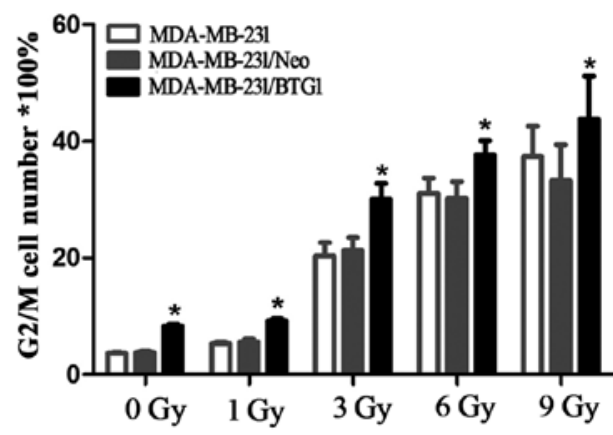

B
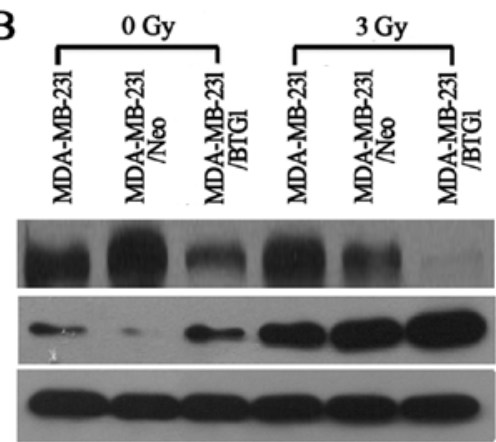
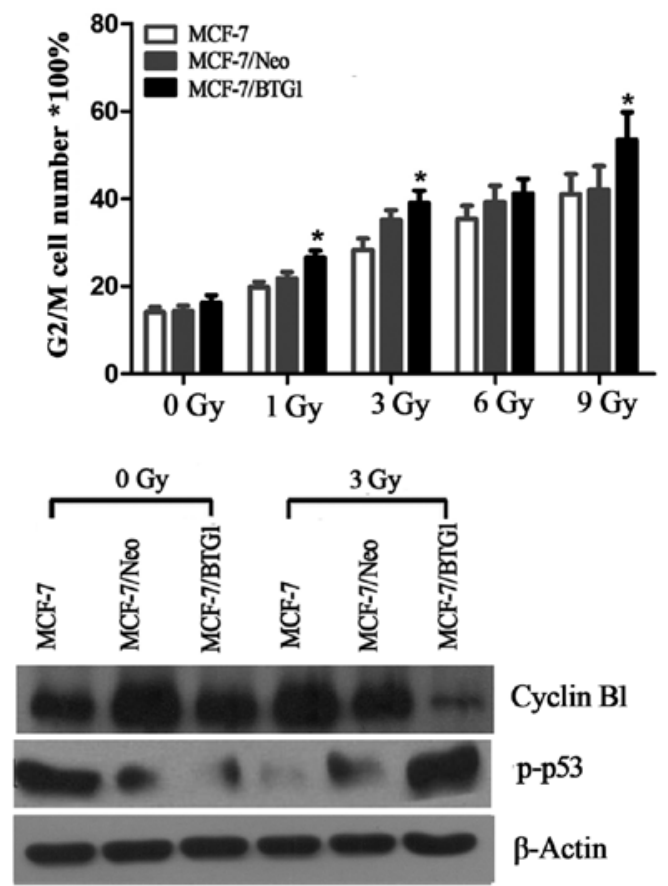

Figure 2. Effects of BTG1 and irradiation on cell cycle distribution and cyclin B1 and p-p53 in the MDA-MB-231 and MCF-7 cells. Cells were exposed to $0,1,3,6$ and $9 \mathrm{~Gy}$ doses of radiation and incubated at $37^{\circ} \mathrm{C}$ for $24 \mathrm{~h}$ post-irradiation. (A) Cells were collected to assay the cell cycle using flow cytometry as described in Materials and methods ( $\left.{ }^{\mathrm{p}} \mathrm{p}<0.05\right)$. (B) Cyclin and p-p53 levels were assessed by western blotting, $\beta$-actin levels served as the loading control. BTG1, B-cell translocation gene 1.

A
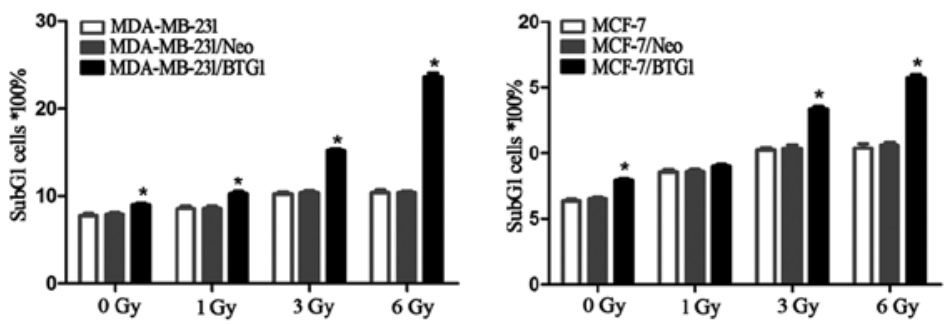

$\mathrm{C}$

B
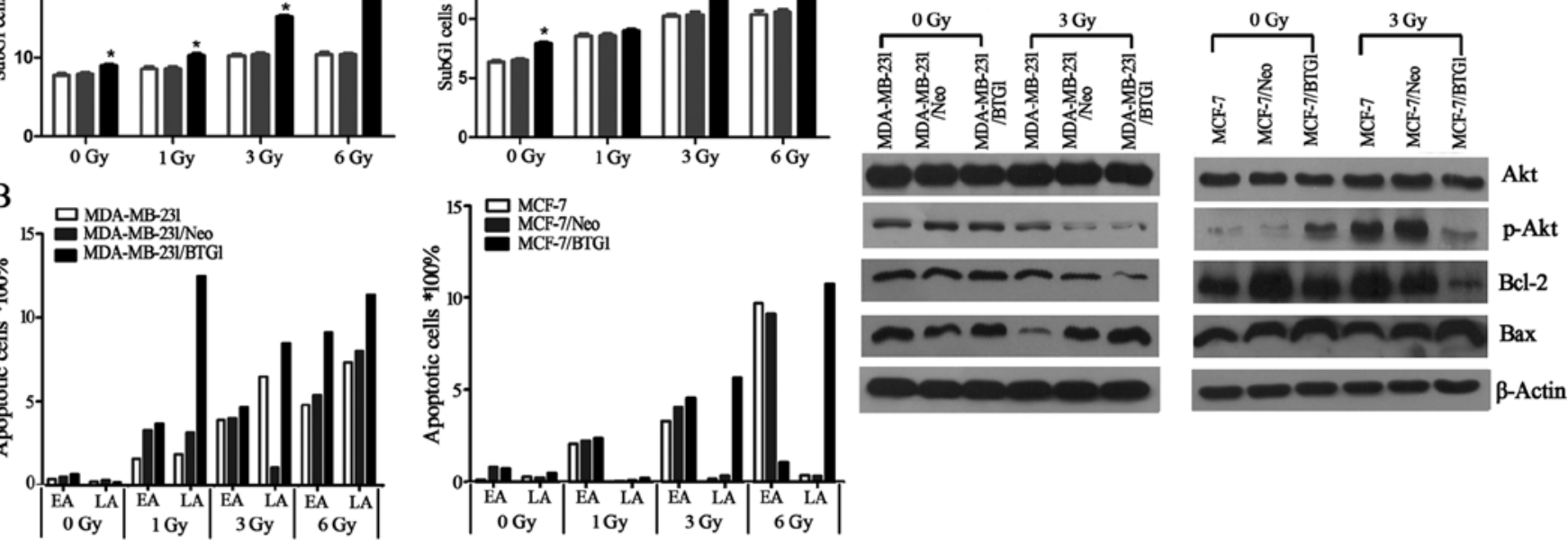

Figure 3. Effects of BTG1 and irradiation on cell apoptosis and apoptosis-related proteins in MDA-MB-231 and MCF-7 cells. Cells were exposed to $0,1,3$ and $6 \mathrm{~Gy}$ doses of radiation and incubated at $37^{\circ} \mathrm{C}$ for $24 \mathrm{~h}$ post-irradiation. (A) The peaks of sub-G1 phase were detected using flow cytometry ("p $<0.05$ ). (B) Annexin V-FITC was used to determine the percentage of apoptosis (EA, early apoptosis; LA, late apoptosis). (C) Apoptosis-related protein levels were assessed by western blotting, $\beta$-actin levels served as the loading control. BTG1, B-cell translocation gene 1 .

percentage of apoptotic cells and the sub-G1 peaks were higher than that in the control cells (Fig. 3A and B), after cells were exposed to irradiation. Next, we detected the expression of anti-apoptotic factor Bcl-2 and pro-apoptotic factors Bax and PI3K/Akt using western blot analysis. As shown in Fig. 3C, overexpression of BTG1 along with irradiation treatment suppressed the PI3K/Akt signaling pathway, downregulated the expression of the anti-apoptotic protein Bcl-2 and upregulated the expression of the pro-apoptotic protein Bax in the MCF-7 and MDA-MB-231 cells. These results indicated that the promotive effect on cell apoptosis by BTG1 along with irradiation is most likely mediated by Bcl-2 and Bax which is regulated by the PI3K/Akt signaling pathway in breast cancer cells. 
A

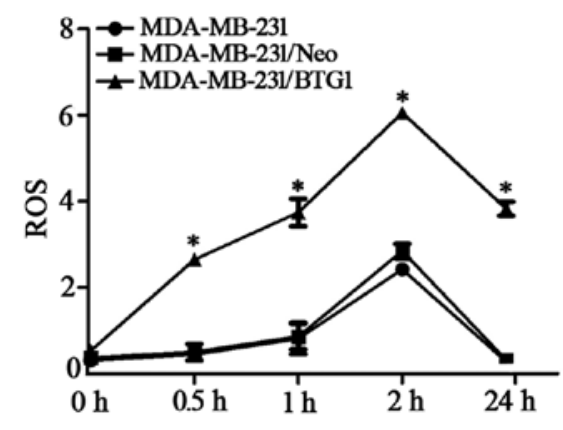

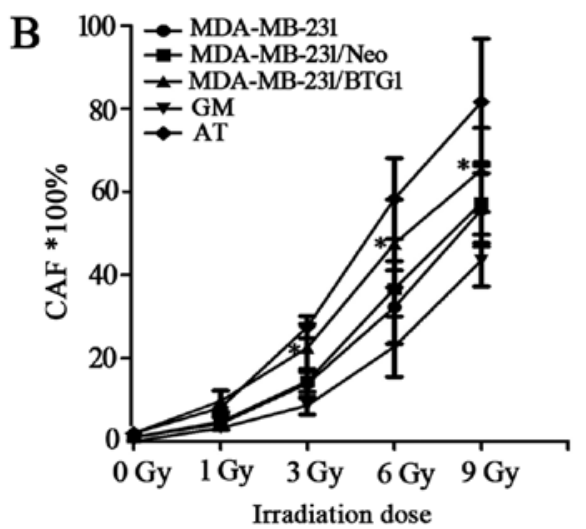

Figure 4. Effects of BTG1 and irradiation on intracellular ROS levels and chromosomal aberrations in MDA-MB-231 cells. (A) Cells were exposed to a 3 Gy dose of radiation and the change in intracellular ROS levels at different observation points was observed ( $\left.{ }^{*} \mathrm{p}<0.05\right)$. (B) Cells were exposed to different doses of radiation and were then treated with $400 \mathrm{ng} / \mathrm{ml}$ colchicine. Chromosome aberrations including dicentric (dic) and centromere ring (r) were stained and counted by microscopic examination in the different groups $(* \mathrm{p}<0.05)$. BTG1, B-cell translocation gene 1 ; ROS, reactive oxygen species.
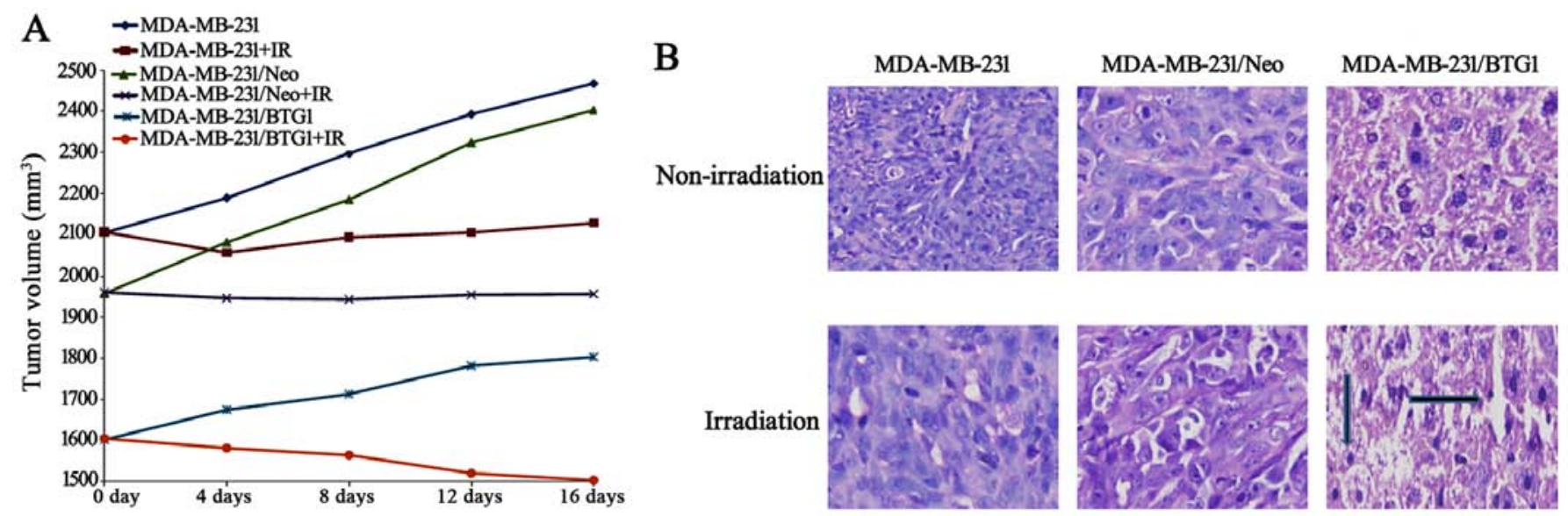

Figure 5. Effects of BTG1 and irradiation on MDA-MB-231 xenografts. The nude mouse models constructed using MDA-MB-231/BTG1 and control cells were randomly selected to accept a $3 \mathrm{~Gy}$ dose of radiation. (A) Tumor diameter was measured every four days (IR, irradiation). (B) H\&E staining showed that tumor density in the MDA-MB-231/BTG1 cell tumors was decreased when compared to the control cell tumors in both the irradiated and non-irradiated groups. H\&E, hematoxylin and eosin. BTG1, B-cell translocation gene 1.

Effects of BTG1 along with irradiation on the intracellular ROS of MDA-MB-231 cells. Intracellular ROS accumulation can activate several pathways important for the induction of apoptosis; thus, we examined the involvement of ROS in BTG1 and irradiation-induced cell death. As shown in Fig. 4A, ROS levels in the BTG1 overexpression group cells were markedly higher than the levels in the control group cells after irradiation of $3 \mathrm{~Gy}$ treatment for $0.5-24 \mathrm{~h}$. This result indicates that upregulation of BTG1 promotes the formation of ROS in breast cancer cells, thereby enhancing cell radiosensitivity.

Effects of irradiation on chromosomal aberrations in MDA-MB-231 cells. MDA-MB-231, MDA-MB-231/Neo, MDA-MB-231/BTG1, GM (normal radiation sensitive) and AT (radiation sensitive) cells were exposed to $0,1,3,6$ and 9 Gy doses of irradiation and chromosomal aberrations were detected. As shown in Fig. 4B, the rates of chromosomal aberrations in the MDA-MB-231/BTG1 cells were significantly higher than those in the MDA-MB-231, MDA-MB-231/Neo and GM cells following irradiation at doses of 3,6 and 9 Gy $(p<0.05)$, yet these rates were lower than those in the AT cells. Those results revealed that BTG1 increased the rate of chromosomal aberrations after breast cancer MDA-MB-231 cells were exposed to moderate- or high-dose irradiation.

Effects of BTG1 on the radiosensitivity of breast cancer in vivo. The three groups of MDA-MB-231 cells were subcutaneously injected into nude mice. Each group consisted of 12 nude mice, and 6 nude mice were randomly selected to accept a 3 Gy dose of radiation. After 16 days of growth, the tumor masses obtained from the irradiated group xenografts were markedly smaller than those from the non-irradiated group (Fig. 5A, $\mathrm{p}<0.05$ ), and the BTG1 overexpression (MDA-MB-231/BTG1) and irradiation group xenografts were smaller than the xenografts from the other groups $(\mathrm{p}<0.05)$. H\&E staining showed that tumor density in the MDA-MB-231/BTG1 cell tumors was decreased when compared to the control cell tumors in both the irradiated and non-irradiated groups (Fig. 5B).

The effect of BTG1 overexpression on tumor angiogenesis in vivo was then assessed using immunohistochemistry. 
A
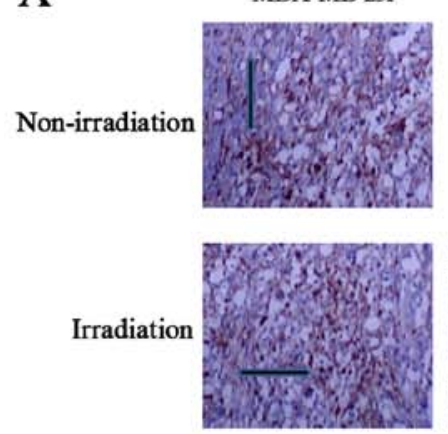

B
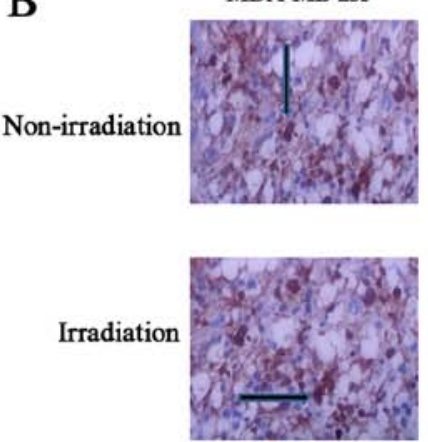

MDA-MB-231/Neo
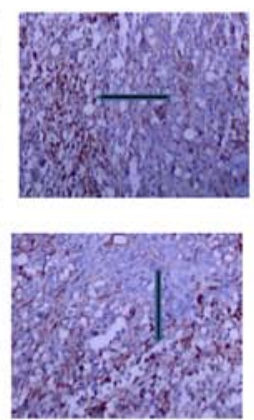

Angiogenesis
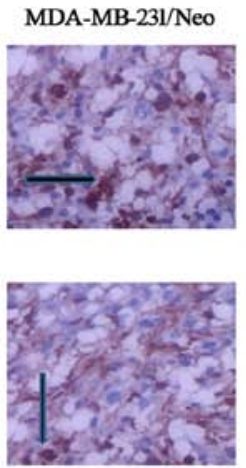

VEGF
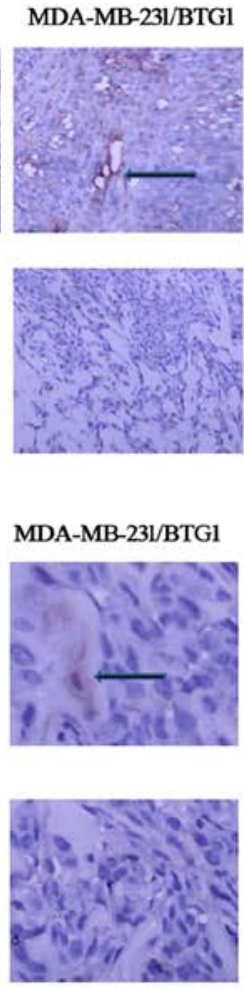

C
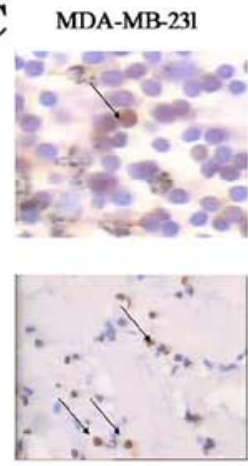

D
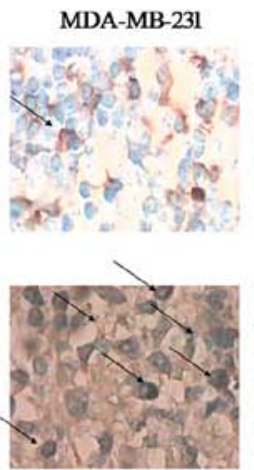

MDA-MB-231/Neo
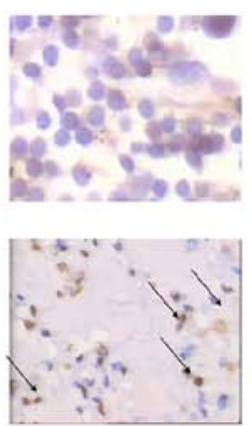

Apoptosis
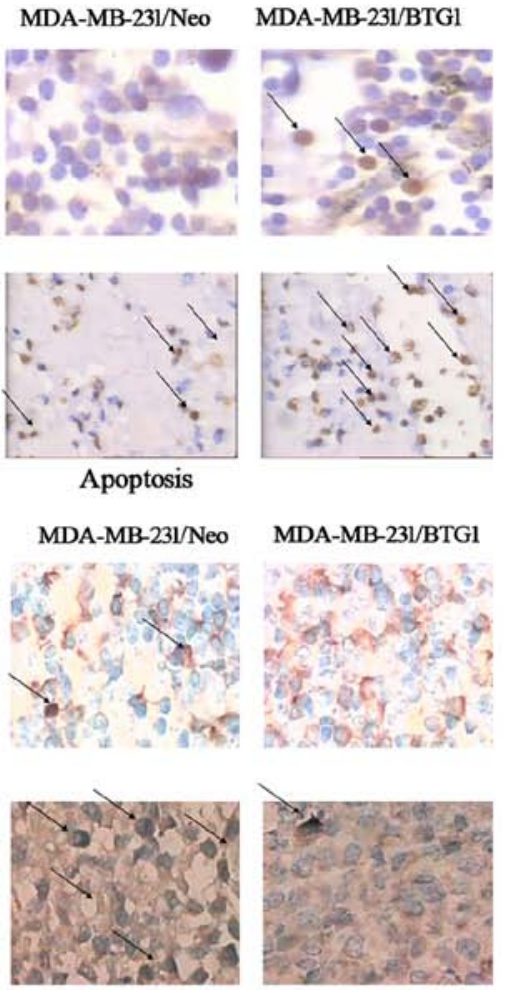

Bcl-2

Figure 6. Histological analysis. (A) Immunohistochemical analysis of angiogenesis using anti-CD31 antibodies. (B) VEGF expression was lower or negative in the MDA-MB-231/BTG1 tumor group whether exposed or not to irradiation. (C) Tumor necrosis and apoptosis were detected using TUNEL staining assay. (D) The immunochemical staining showed that overexpression of BTG1 along with irradiation treatment downregulated the expression of anti-apoptotic protein Bcl-2. VEGF, vascular endothelial growth factor.

Anti-CD31 antibodies were utilized to detect CD31 levels, which were used to reflect the level of vascular endothelial proliferation. As shown in Fig. 6A, the number of tumor microvessels in the MDA-MB-231 and MDA-MB-231/Neo tumor groups was 13 and 11, respectively, and significantly more than the MDA-MB-231/BTG1 tumor group ( $\mathrm{p}<0.05)$. In contrast, the number of tumor microvessels in the MDA-MB231/BTG1 nude mice irradiated with 3 Gy X-ray was 2-3, which was less than that in the other groups $(\mathrm{p}<0.05)$. In addition, VEGF expression was lower or negative in the MDA-MB-231/ BTG1 tumor group whether irradiation or not (Fig. 6B). These results suggest that overexpression of BTG1 along with irradiation may inhibit angiogenesis through the downregulation of VEGF expression, thereby inhibiting tumor metastasis.

Next, we assessed tumor necrosis and apoptosis by TUNEL staining assay. As shown in Fig. 6C, various cells exhibited nuclear pyknosis and fragmented, irregular, inconsistency in size and brownish yellow staining indicating cell apoptosis. Thus, several visible sporadic apoptotic cells were presented in the MDA-MB-231, MDA-MB-231/Neo tumor tissue. In contrast, a high number of apoptotic cells was observed in the MDA-MB-231/BTG1 tumor tissue. The immunochemical staining showed that overexpression of BTG1 along with the irradiation treatment downregulated the expression of the anti-apoptotic protein Bcl-2 (Fig. 6D).

Clearly, these in vivo results strongly confirm the effect observed in vitro indicating that BTG1 plays an important role in breast cancer cell growth and radiosensitivity.

\section{Discussion}

Radiotherapy is arguably the most important treatment for cancer, particularly for loco-regional tumors without metastasis. Ionizing radiation is used to treat nearly all types of solid tumors, yet to varying degrees of success $(17,18)$. The reasons for radiotherapy failure are multiple and varied. Cells in different cell cycle phases have variant radiosensitivity. In general, cells in the G0/G1 phase have a certain radiation resistance and the highest sensitivity exists for cells in the boundary of the G0/G1 and S phase. When cells enter the $\mathrm{S}$ phase, the radiation resistance increases gradually and reaches the highest at the late $\mathrm{S}$ phase, yet it is decreased in the G2/M phase (19). In the present study, our results showed that overexpression of BTG1 induced G2/M phase arrest, which increased the ionizing radiosensitivity of breast cancer cells.

The regulation of the cell cycle is one of the main influencing mechanisms of cellular radiosensitivity. Cell cycle regulation after ionizing radiation occurs in the control points of G1/S and G2/M phase, where DNA damage repair-related proteins are activated preventing the unrepaired DNA into S phase (20). The p53 gene is an important effector in the human cell cycle checkpoint, and can be phosphorylated by DNA damage signal caused by irradiation. Then p53 starts p 21 gene transcription, promotes the expression of $\mathrm{p} 21^{\mathrm{CIP} / \mathrm{WAF} 1}$, and inhibits the activity of CDK2 to prevent cells from entering the $\mathrm{S}$ phase $(21,22)$. p53-mutant cells show G2 phase arrest in DNA damage repair, and the length of G2 phase arrest is 
proportional to the degree of DNA damage. G2 phase arrest leads to an increase in cell death and enhanced cell radiosensitivity in theory. Our results indicated that overexpression of BTG1 increased p-p53 expression after cells were exposed to irradiation, which enhanced breast cancer cell radiosensitivity. Cyclin B1 expression is minimal at the initiation of the $S$ phase and peaks at the G2-M border; this peak in cyclin B1 activity is required for cells entering mitosis (23). Thus, overexpression of cyclin B1 leads to G2/M conversion, and even leads to uncontrolled cell proliferation and malignant transformation (24). Phosphorylation of cyclin B1 inhibits Cdk1 activation through the $\mathrm{p} 21 / \mathrm{Gadd} 45 / \mathrm{Cdk} 1$ pathway activated by $\mathrm{p} 53$ which result in $\mathrm{G} 2 / \mathrm{M}$ phase arrest (25). Cyclin B1 overexpression is also closely related to the pathological type of tumor, tumor metastasis and prognosis, and causes tumor radiation resistance (26). In the present study, we found that overexpression of BTG1 decreased cyclin B1 expression after breast cancer cells were exposed to irradiation. These data indicated that BTG1 participates in $\mathrm{G} 2 / \mathrm{M}$ phase checkpoint regulation through inhibition of cyclin B1 expression and enhances breast cancer cell radiosensitivity.

Cell apoptosis is also one of the molecular mechanisms that improves tumor cell radiosensitivity (27). The Bcl-2 family of proteins serves as critical regulators of apoptosis that can be identified as anti-apoptotic proteins. The antiapoptotic Bcl-2/Bcl-xL and pro-apoptotic Bax/Bak proteins can both negatively and positively regulate events in apoptotic cell death through modulation of cytochrome $c$ release (28). The PI3K/Akt pathway is an important intracellular signaling pathway in the regulation of cell growth, survival, adhesion and migration, particularly during cancer progression, metastasis and radioresistance $(29,30)$. In the present study, we demonstrated that overexpression of BTG1 reduced p-Akt, Bcl-2 and increased Bax expression after breast cancer cells were exposed to irradiation. These results indicate that the PI3K/Akt pathway mediates BTG1 upregulation of breast cancer cell radiosensitivity.

Recent studies suggest that reactive oxygen species (ROS) may play an important role during the induction of apoptosis (31). Many stimuli such as tumor necrosis factor- $\alpha$, anticancer drugs and chemopreventive agents stimulate cells to produce ROS (32-35). Intracellular ROS can also be induced by irradiation and serve as major mediators of radiation damage (36). When ROS generation exceeds the cellular antioxidant defenses, cell damage ensues $(37,38)$. In the present study, we found that overexpression of BTG1 along with irradiation treatment led to significant elevation in the levels of intracellular ROS and cell apoptosis rates, which further indicated that upregulation of BTG1 promoted the formation of ROS in breast cancer cells, thereby enhancing cell radiosensitivity.

Importantly, the finding that BTG1 promoted ionizing radiosensitivity of breast cancer cells in vitro was confirmed in our animal model. After the nude mouse model was treated with irradiation, the volume of established BTG1 overexpression xenografts was smaller than the volume in the vector control group, and the immunochemical staining showed that the levels of Bcl-2 and VEGF expression were lower than those in the control group. Next, we found that the proportion of apoptotic cells was increased and the tumor angiogenesis was reduced in the experimental group xenografts, as detected by TUNEL and immunohistochemical analysis.

In summary, the present study investigating the role of BTG1 in breast cancer cell radiosensitivity demonstrated that the protein can enhance cell radiosensitivity. The mechanism of action may be related to cell cycle regulation, $\mathrm{G} 2 / \mathrm{M}$ phase arrest, ROS formation, promotion of cell apoptosis and inhibition of tumor angiogenesis. These findings provide new insight into the role of BTG1 in breast cancer radiosensitivity and may have important implication for the development of radiation therapy for breast cancer.

\section{Acknowledgements}

The present study was supported by the National Natural Science Foundation of China (nos. 81502758 and 81302383), the Science and Technology Foundation of Suzhou (nos. SYS201215 and SYS201417), and a Project Funded by the Priority Academic Program Development of Jiangsu Higher Education Institutions (PAPD).

\section{References}

1. Printz C: American Cancer Society reports progress in reducing cancer deaths: However, some groups still lag behind this trend. Cancer 117: 4573-4574, 2011.

2. Jemal A, Siegel R, Xu J and Ward E: Cancer statistics, 2010. CA Cancer J Clin 60: 277-300, 2010.

3. DeSantis C, Ma J, Bryan L and Jemal A: Breast cancer statistics, 2013. CA Cancer J Clin 64: 52-62, 2014.

4. Pekkola-Heino K, Servomaa K, Kiuru A and Grenman R: Increased radiosensitivity is associated with p53 mutations in cell lines derived from oral cavity carcinoma. Acta Otolaryngol 116: 341-344, 1996.

5. Huerta S, Gao X, Dineen S, Kapur P, Saha D and Meyer J: Role of p53, Bax, p21, and DNA-PKes in radiation sensitivity of HCT-116 cells and xenografts. Surgery 154: 143-151, 2013.

6. Berthet C, Guéhenneux F, Revol V, Samarut C, Lukaszewicz A, Dehay C, Dumontet C, Magaud JP and Rouault JP: Interaction of PRMT1 with BTG/TOB proteins in cell signalling: Molecular analysis and functional aspects. Genes Cells 7: 29-39, 2002.

7. Rouault JP, Rimokh R, Tessa C, Paranhos G, Ffrench M, Duret L, Garoccio M, Germain D, Samarut J and Magaud JP: BTG1, a member of a new family of antiproliferative genes. EMBO J 11: 1663-1670, 1992.

8. Matsuda S, Rouault J, Magaud J and Berthet C: In search of a function for the TIS21/PC3/BTG1/TOB family. FEBS Lett 497 : 67-72, 2001.

9. Bakker WJ, Blázquez-Domingo M, Kolbus A, Besooyen J, Steinlein P, Beug H, Coffer PJ, Löwenberg B, von Lindern M and van Dijk TB: FoxO3a regulates erythroid differentiation and induces BTG1, an activator of protein arginine methyl transferase 1. J Cell Biol 164: 175-184, 2004.

10. Corjay MH, Kearney MA, Munzer DA, Diamond SM and Stoltenborg JK: Antiproliferative gene BTG1 is highly expressed in apoptotic cells in macrophage-rich areas of advanced lesions in Watanabe heritable hyperlipidemic rabbit and human. Lab Invest 78: 847-858, 1998.

11. Rodier A, Marchal-Victorion S, Rochard P, Casas F, CassarMalek I, Rouault JP, Magaud JP, Mason DY, Wrutniak C and Cabello G: BTG1: A triiodothyronine target involved in the myogenic influence of the hormone. Exp Cell Res 249: 337-348, 1999.

12. Lee H, Cha S, Lee MS, Cho GJ, Choi WS and Suk K: Role of antiproliferative B cell translocation gene-1 as an apoptotic sensitizer in activation-induced cell death of brain microglia. $\mathbf{J}$ Immunol 171: 5802-5811, 2003.

13. Sheng SH, Zhao CM and Sun GG: BTG1 expression correlates with the pathogenesis and progression of breast carcinomas. Tumour Biol 35: 3317-3326, 2014.

14. Li W, Zou ST, Zhu R, Wan JM, Xu Y and Wu HR: B-cell translocation 1 gene inhibits cellular metastasis-associated behavior in breast cancer. Mol Med Rep 9: 2374-2380, 2014. 
15. Wu X, Ding N, Hu W, He J, Xu S, Pei H, Hua J, Zhou G and Wang J: Down-regulation of BTG1 by miR-454-3p enhances cellular radiosensitivity in renal carcinoma cells. Radiat Oncol 9: 179,2014

16. Zhu R, Zou ST, Wan JM, Li W, Li XL and Zhu W: BTG1 inhibits breast cancer cell growth through induction of cell cycle arrest and apoptosis. Oncol Rep 30: 2137-2144, 2013.

17. Peters LJ, Withers HR, Thames HD Jr and Fletcher GH: Tumor radioresistance in clinical radiotherapy. Int J Radiat Oncol Biol Phys 8: 101-108, 1982.

18. Deacon J, Peckham MJ and Steel GG: The radioresponsiveness of human tumours and the initial slope of the cell survival curve. Radiother Oncol 2: 317-323, 1984.

19. Wyld L, Smith O, Lawry J, Reed MW and Brown NJ: Cell cycle phase influences tumour cell sensitivity to aminolaevulinic acidinduced photodynamic therapy in vitro. Br J Cancer 78: 50-55, 1998.

20. Efeyan A and Serrano M: p53: Guardian of the genome and policeman of the oncogenes. Cell Cycle 6: 1006-1010, 2007.

21. Duan X, Zhang H, Liu B, Li XD, Gao QX and Wu ZH: Apoptosis of murine melanoma cells induced by heavy-ion radiation combined with Tp53 gene transfer. Int J Radiat Biol 84: 211-217, 2008.

22. Hill R, Bodzak E, Blough MD and Lee PW: p53 binding to the p21 promoter is dependent on the nature of DNA damage. Cell Cycle 7: 2535-2543, 2008.

23. Hwang A, McKenna WG and Muschel RJ: Cell cycle-dependent usage of transcriptional start sites. A novel mechanism for regulation of cyclin B1. J Biol Chem 273: 31505-31509, 1998.

24. Ozeki M, Tamae D, Hou DX, Wang T, Lebon T, Spitz DR and Li JJ: Response of cyclin B1 to ionizing radiation: Regulation by NF-kappaB and mitochondrial antioxidant enzyme MnSOD. Anticancer Res 24: 2657-2663, 2004.

25. Raj D, Liu T, Samadashwily G, Li F and Grossman D: Survivin repression by $\mathrm{p} 53, \mathrm{Rb}$ and $\mathrm{E} 2 \mathrm{~F} 2$ in normal human melanocytes. Carcinogenesis 29: 194-201, 2008.

26. Hassan KA, Ang KK, El-Naggar AK, Story MD, Lee JI, Liu D, Hong WK and Mao L: Cyclin B1 overexpression and resistance to radiotherapy in head and neck squamous cell carcinoma. Cancer Res 62: 6414-6417, 2002.

27. Lee CH, Jeon YT, Kim SH and Song YS: NF-kappaB as a potential molecular target for cancer therapy. Biofactors 29: 19-35, 2007.
28. Cory S, Huang DC and Adams JM: The Bcl-2 family: Roles in cell survival and oncogenesis. Oncogene 22: 8590-8607, 2003.

29. Chang L, Graham PH, Hao J, Ni J, Bucci J, Cozzi PJ, Kearsley JH and Li Y: Acquisition of epithelial-mesenchymal transition and cancer stem cell phenotypes is associated with activation of the $\mathrm{PI} 3 \mathrm{~K} / \mathrm{Akt} / \mathrm{mTOR}$ pathway in prostate cancer radioresistance. Cell Death Dis 4: e875, 2013.

30. Chang L, Graham PH, Hao J, Bucci J, Cozzi PJ, Kearsley JH and $\mathrm{Li}$ Y: Emerging roles of radioresistance in prostate cancer metastasis and radiation therapy. Cancer Metastasis Rev 33: 469-496, 2014.

31. Schulz JB, Bremen D, Reed JC, Lommatzsch J, Takayama S, Wüllner U, Löschmann PA, Klockgether T and Weller M: Cooperative interception of neuronal apoptosis by BCL-2 and BAG-1 expression: Prevention of caspase activation and reduced production of reactive oxygen species. J Neurochem 69: 2075-2086, 1997.

32. Goossens V, De Vos K, Vercammen D, Steemans M, Vancompernolle K, Fiers W, Vandenabeele P and Grooten J: Redox regulation of TNF signaling. Biofactors 10: 145-156, 1999.

33. Simizu S, Takada M, Umezawa K and Imoto M: Requirement of caspase-3(-like) protease-mediated hydrogen peroxide production for apoptosis induced by various anticancer drugs. J Biol Chem 273: 26900-26907, 1998.

34. Chan WH, Yu JS and Yang SD: PAK2 is cleaved and activated during hyperosmotic shock-induced apoptosis via a caspasedependent mechanism: Evidence for the involvement of oxidative stress. J Cell Physiol 178: 397-408, 1999.

35. Vercammen D, Brouckaert G, Denecker G, Van de Craen M, Declercq W, Fiers W and Vandenabeele P: Dual signaling of the Fas receptor: Initiation of both apoptotic and necrotic cell death pathways. J Exp Med 188: 919-930, 1998.

36. Mikkelsen RB and Wardman P: Biological chemistry of reactive oxygen and nitrogen and radiation-induced signal transduction mechanisms. Oncogene 22: 5734-5754, 2003

37. Gamaley IA and Klyubin IV: Roles of reactive oxygen species: Signaling and regulation of cellular functions. Int Rev Cytol 188: 203-255, 1999.

38. Cerutti PA: Prooxidant states and tumor promotion. Science 227: 375-381, 1985. 